\title{
A RADIAL UNIQUENESS THEOREM FOR SOBOLEV FUNCTIONS
}

\author{
P. KOSKELA
}

\begin{abstract}
We show that continuous functions $u$ in the Sobolev space $W_{p}^{1}(B), 1<p \leqslant n$, which have the limit zero in a certain weak sense in a set of positive $p$-capacity on $\partial B$ with

$$
\int_{B_{\varepsilon}}|\nabla u|^{p} d x \leqslant C \varepsilon^{p}\left(\log \left[\frac{1}{\varepsilon}\right]\right)^{p-1},
$$

where $B$ is the open unit ball of $\mathbb{R}^{n}$ and

for $0<\varepsilon<\frac{1}{2}$, are identically zero.

$$
B_{\varepsilon}=\{x \in B:|u(x)|<\varepsilon\}
$$

Conversely, we produce for each $1<p \leqslant n$ and each positive $\delta$ a non-constant function $u$ in $W_{p}^{1}(B)$, continuous in $\bar{B}$, and a compact set $E \subset \partial B$ of positive $p$-capacity such that $u=0$ in $E$ and the above inequality holds with exponent $p-1+\delta$.
\end{abstract}

\section{Introduction}

In this note we address the following question. Suppose that $u$ is a continuous function in the Sobolev space $W_{p}^{1}(B), 1<p \leqslant n$, where $B$ is the open unit ball of $\mathbb{R}^{n}$ (then $u$ has radial limits except for a set in $\partial B$ of Bessel $p$-capacity zero, see, for example, $[11,8])$. Assume that $u$ approaches zero in the weak sense for each $x \in E \subset$ $\partial B$ of positive $p$-capacity. Here we declare that $u$ approaches zero in the weak sense if for any rectifiable curve $\gamma$ in $B$ terminating at $x$ there is a sequence of points in $\gamma$ for which $u$ tends to zero. We ask: under which conditions does it follow that $u$ is the constant function zero?

This question has been studied by several authors for analytic functions $f$ of the unit disc $D$ with $\left|f^{\prime}\right| \in L^{2}(D)$. Note that in this situation the coordinate functions of $f$ belong to $W_{2}^{1}(D)$, and that $f$ approaches zero in the above weak sense if $f$ has the radial limit zero for each $x \in E$; see Section 2. A. Beurling [2] established that there are no bounded univalent functions with the radial limit zero in a set of positive 2capacity. L. Carleson [3] produced examples of non-constant analytic functions of $D$ with $\left|f^{\prime}\right| \in L^{2}(D)$ which have the radial (hence also non-tangential and weak) limit zero in a set $E$ of positive 2-capacity, and studied the properties of the associated sets $E$. M. Tsuji [12] verified that such an $f$ is identically zero provided

$$
\iint_{D_{\varepsilon}}\left|f^{\prime}\right|^{2} d x d y \leqslant C \varepsilon^{2}
$$

for all $0<\varepsilon<1$, where $D_{\varepsilon}=\{z \in D:|f(z)|<\varepsilon\}$. Recently, E. Villamor [14] extended this result by showing that it suffices to assume the integral in (1) to be of the order 
of $o\left(\varepsilon^{2} \log [1 / \varepsilon]\right)$. We establish that this phenomenon is not characteristic of analytic functions but merely a property of continuous Sobolev functions. Let us introduce some notation. We write $u \in A C L^{p}(B)$ if $u \in W_{p}^{1}(B)$ is continuous (we advise the reader that our definition for $A C L^{p}(B)$ is not standard), and set

$$
B_{\varepsilon}=\{x \in B:|u(x)|<\varepsilon\}
$$

for $\varepsilon>0$. Then we have the following.

THEOREM A. Let $u \in A C L^{p}(B), 1<p \leqslant n$, approach zero in the weak sense for a set $E \subset \partial B$ of positive p-capacity. If there is a constant $C$ such that

$$
\int_{B_{\varepsilon}}|\nabla u|^{p} d x \leqslant C \varepsilon^{p}\left(\log \left[\frac{1}{\varepsilon}\right]\right)^{p-1}
$$

for all $0<\varepsilon<\frac{1}{2}$, then $u$ is identically zero.

As the following result indicates, our assertion is essentially sharp.

THEOREM B. For each $1<p \leqslant n$ and any $\delta>0$, there is a non-constant function $u \in A C L^{p}(B)$, continuous in the closed unit ball, and a compact set $E \subset \partial B$ of positive p-capacity with $u=0$ in $E$ such that

$$
\int_{B_{\varepsilon}}|\nabla u|^{p} d x \leqslant C \varepsilon^{p}\left(\log \left[\frac{1}{\varepsilon}\right]\right)^{p-1+\delta}
$$

for all $0<\varepsilon<\frac{1}{2}$.

It remains open whether the given bound in Theorem A is also essentially sharp for analytic functions of the unit disc. We wish to point out that Theorem A extends Villamor's result even when $p=2$ and $f$ is analytic (apply Theorem $\mathrm{A}$ to the coordinate functions of $f$ ), and that the proof of Theorem A avoids the complicated calculations in [14].

J. A. Jenkins [5] has recently established results related to Theorem A for meromorphic functions for $p=n=2$.

\section{Preliminaries}

We denote the p-modulus of a curve family $\Gamma$ by $\bmod _{p}(\Gamma)$. Thus

$$
\bmod _{p}(\Gamma)=\inf _{\rho} \int_{\mathbb{R}^{n}} \rho^{p} d x,
$$

where the infimum is taken over all non-negative Borel-measurable $\rho$ such that

$$
\int_{\gamma} \rho d s \geqslant 1
$$

for each locally rectifiable $\gamma \in \Gamma$; see $[13,6.1]$. 
Next, the $p$-capacity of $E, F \subset \bar{D}$, for a domain $D$ and a pair $E, F$ of disjoint, nonempty, compact sets is defined by

$$
\operatorname{cap}_{p}(E, F ; D)=\inf _{u \in L} \int_{D}|\nabla u|^{p} d x,
$$

where the infimum is taken over all functions in the class

$$
L=L(E, F ; D)=\left\{u \in L_{p}^{1}(D) \cap C(D \cup E \cup F):\left.u\right|_{E} \leqslant 0,\left.u\right|_{F} \geqslant 1\right\} .
$$

Here $L_{p}^{1}(D)$ denotes the Sobolev space of measurable functions $u: D \rightarrow \overline{\mathbb{R}}$ satisfying $\int_{D}|\nabla u|^{p} d x<\infty$, where $\nabla u$ represents the distributional gradient of $u$. Recall that $W_{p}^{1}(D)=L^{p}(D) \cap L_{p}^{1}(D)$ and that $L_{p}^{1}(D) \subset W_{p}^{1}(D)$ if $\partial D$ is sufficiently smooth. In particular, this is true for $B$.

We say that a compact set $E$ is of $p$-capacity zero if $\operatorname{cap}_{p}(E, \partial D ; D)=0$ for some bounded (and hence for each bounded) domain $D$ containing $E$. We remind the reader that this is the case (see, for example, [15]) if the $p$-modulus of the family of curves in $\mathbb{R}^{n}$ joining $E$ to the boundary of some open ball $B^{\prime}$ is zero.

We begin by showing that for functions in $A C L^{n}(B)$, radial limits are limits in the weak sense. This justifies the comment made in the introduction.

LeMMA 2.1. Let $u \in A C L^{n}(B)$ have the radial limit zero in a compact set $E \subset \partial B$. Then $u$ has the limit zero in the weak sense in $E$.

Proof. Let $u$ and $E$ be as above, and fix a curve $\gamma$ terminating at $x \in E$. If $u$ does not tend to zero along any sequence in $\gamma$, we find $\varepsilon>0$ and $r>0$ such that $|u|>2 \varepsilon$ in $K_{1}=\gamma \cap B(x, r)$ and $|u|<\varepsilon$ in $K_{2}=J \cap B(x, r)$, where $J$ is the radius containing $x$. Thus

$$
\operatorname{cap}_{n}\left(K_{1}, K_{2} ; B\right) \leqslant \int_{B}|\nabla u|^{n} d x \varepsilon^{-n}<\infty,
$$

which is a contradiction since both $K_{1}$ and $K_{2}$ connect $x$ to $\partial B(x, r)$; see [13, 10.12].

If $h$ is an increasing homeomorphism of $(0, \infty)$, then the $h$-Hausdorff measure of a set $E \subset \mathbb{R}^{n}$ is defined by

$$
H_{h}(E)=\sup _{\delta>0} H_{h}^{\delta}(E)
$$

where

$$
H_{h}^{\delta}(E)=\inf \left\{\sum_{j=1}^{\infty} h\left(r_{j}\right): r_{j} \leqslant \delta, E \subset \bigcup_{j=1}^{\infty} B\left(x_{j}, r_{j}\right)\right\} .
$$

We record Theorem 7.1 in [7, p. 133].

LEMMA 2.2. If $H^{h}(E)>0$ and

$$
\int_{0}^{1 / 2}\left(h(t) t^{p-n}\right)^{1 /(p-1)} t^{-1} d t<\infty
$$

then $\operatorname{cap}_{p}(E)>0$.

Next, we recall the generalized Cantor construction. Let $\left(l_{j}\right)$ be a sequence of positive numbers decreasing to zero so that $2 l_{j+1}<l_{j}$ for each $j$. Set $I_{\mathbf{0 , 1}}=\left[0, l_{1}\right]$, and 
let $I_{1,1}$ and $I_{1,2}$ be the intervals $\left[0, l_{2}\right]$ and $\left[l_{1}-l_{2}, l_{1}\right]$, respectively. Assuming that the intervals $I_{k-1,1}, \ldots, I_{k-1,2^{k-1}}$ have been selected, we define $I_{k, 1}, \ldots, I_{k, 2^{k}}$ by deleting from the middle of each of the intervals $I_{k-1, j}$ an interval of length $l_{k}-2 l_{k+1}$.

Write $F_{j}=\bigcup_{i=1}^{2^{j}} I_{j, i}$, set $E_{j}=F_{j}^{n-1}$ (the product of $F_{j}$ with itself $(n-1)$ times), and define $E=\bigcap_{j=1}^{\infty} E_{j}$.

We shall deduce Theorem $B$ from the following.

Lemma 2.3. For each $1<p \leqslant n$ and each $\delta>0$, there is a compact set $E \subset \partial B$ of positive p-capacity with

$$
|E(r)| \leqslant C r^{p}\left(\log \left[\frac{1}{r}\right]\right)^{p-1+\delta}
$$

for all $0<r<\frac{1}{2}$, where $E(r)=\{x$ : dist $(x, E)<r\}$.

Proof. Fix $\delta>0$. We may assume that $\delta<1$. For $1<p<n$, let $E$ be the generalized Cantor set in $\mathbb{R}^{n-1}$ obtained from the sequence

$$
l_{j}=2^{-a\left(j+j_{0}\right)}\left(j+j_{0}\right)^{b},
$$

where $a=(n-1) /(n-p), b=\delta+(p-1) /(n-p)$, and $j_{0}$ is selected sufficiently large to ensure the inequality $2 l_{j+1}<l_{j}$ for all $j$. Next, for $p=n$, replace $l_{\text {, with }}$

$$
l_{j}^{\prime}=\exp \left(-2^{(j+1)(n-1) /(n-1+\delta)}\right)
$$

We show that $E$ has the indicated properties; a subset of $\partial B$ with these properties is then clearly obtained as an appropriate bi-Lipschitz image of $E$.

First let $1<p<n$, and define

$$
h(t)=t^{n-p}\left(\log \left[\frac{1}{t}\right]\right)^{1-p-(n-p) \delta}
$$

for $0<t<1$. Now

$$
l_{j}^{n-p}=2^{-(n-1)\left(j+j_{0}\right)}\left(j+j_{0}\right)^{p-1+\delta(n-p)},
$$

and thus a straightforward calculation verifies that

$$
h\left(l_{j}\right) \geqslant 2^{-(n-1) j} / C
$$

for some constant $C$ for each $j$. As is well known (see, for example, [1, p. 899]), this gives $H^{n}(E)>0$. Since

$$
\int_{0}^{1 / 2}\left(h(t) t^{p-n}\right)^{1 /(p-1)} t^{-1} d t<\infty,
$$

Lemma 2.1 permits us to conclude $\operatorname{cap}_{p}(E)>0$.

We are left with the estimate on $|E(r)|$. Let $0<r<\frac{1}{2}$. Then there exist a constant $C$ depending only on $p, n, \delta$ and a positive integer $j$ such that

$$
r \leqslant l_{j} \leqslant C r .
$$

Now $E(r)$ is contained in $E_{j}(r)$, and we have

$$
|E(r)| \leqslant\left|E_{j}(r)\right| \leqslant\left(3 l_{j}\right)^{n} 2^{(n-1) j} \leqslant 3^{n} l_{j}^{p}\left(j+j_{0}\right)^{p-1+(n-p) \delta} .
$$


A simple but tedious calculation verifies that

$$
C_{1}\left(\log \frac{1}{r}\right)^{p-1+(n-p) \delta} \geqslant\left(j+j_{0}\right)^{p-1+(n-p) \delta}
$$

for a constant $C_{1}$ independent of $r$, and hence

$$
|E(r)| \leqslant C_{2} r^{p}\left(\log \frac{1}{r}\right)^{p-1+(n-p) \delta}
$$

for some constant $C_{2}$ independent of $r$, as desired.

Next, let $p=n$, and define $h(t)=2^{n-1}(\log [1 / t])^{-n+1-\delta}$. Then $h\left(l_{j}^{\prime}\right)=2^{-(n-1) \jmath}$ and we conclude, reasoning as above, that $\operatorname{cap}_{n}(E)>0$. Now fix $0<r<\frac{1}{2}$. We may assume that $r \leqslant \exp (-4)$. Then there is a positive integer $j \geqslant 2$ with $l_{j}<r \leqslant l_{j-1}$, and we obtain

$$
|E(r)| \leqslant\left|E_{j}(r)\right| \leqslant 2^{(n-1) j}(3 r)^{n} \leqslant 3^{n} r^{n}\left(\log \left[\frac{1}{r}\right]\right)^{n-1+\delta} .
$$

The proof is complete.

\section{Proofs of Theorems $A$ and $B$}

First we prove Theorem A.

Proof of Theorem A. Assume that $u$ is not identically zero, and fix a closed ball $B_{0}$ with non-empty interior so that $|u| \geqslant 2^{-j_{0}}, j_{0}>0$, in $B_{0}$. Write

$$
B_{j}=\left\{x \in B: 2^{-j-1} \leqslant|u(x)|<2^{-j}\right\}
$$

for $j=j_{0}, j_{0}+1, \ldots$ Next, define $\rho(x)=|\nabla u(x)|(j \log j)^{-1} 2^{j}$ when $x \in B_{j}$, and set $\rho$ to be zero elsewhere in $\mathbb{R}^{n}$. Then $\rho$ is Borel-measurable (see, for example, $[13,26.4]$ ) and

$$
\begin{aligned}
\int_{B}|\rho|^{p} d x & \leqslant \sum_{j_{0}}^{\infty}(j \log j)^{-p} 2^{j p} \int_{B_{j}}|\nabla u|^{p} d x \\
& \leqslant C(\log 2)^{p-1} \sum_{j_{0}}^{\infty} j^{-1}(\log j)^{-p}<\infty .
\end{aligned}
$$

Fix a rectifiable curve $\gamma$ joining $\partial B_{0}$ to $E$ in $B$, and assume that $u$ is absolutely continuous on each closed subcurve of $\gamma$. Then $u$ approaches zero for some sequence of points in $\gamma$ and, in particular, there is a subcurve $\gamma_{j} \subset B_{j}$ of $\gamma$ for each $j=j_{0}$, $j_{0}+1, \ldots$, with

$$
\int_{\gamma_{j}}|\nabla u| d s \geqslant 2^{-j-1}
$$

Thus

$$
\int_{\gamma} \rho d s \geqslant \frac{1}{2} \sum_{j_{0}}^{\infty} j^{-1}(\log j)^{-1}=\infty .
$$

Finally, $p$-almost every curve in $B$ is rectifiable, and by Fuglede's theorem [13, 28.2], the $p$-modulus of the curves $\gamma$, for which $u$ fails to be absolutely continuous on some closed subcurve, is zero. Hence we infer that the $p$-modulus of the curve family $\Gamma$ joining $E$ to $B_{0}$ in $B$ is zero. As is well known, this yields that the $p$-modulus of the 
curve family joining $E$ to $B_{0}$ in $\mathbb{R}^{n}$ is zero. Indeed, by a simple reflection argument, this holds for the corresponding curve family, say, in $B(0,2)$, and consequently by [6] the $p$-modulus of the curve family joining $E$ to $B_{0}$ in $\mathbb{R}^{n}$ is zero. Hence the $p$-capacity of $E$ is zero, as desired.

Proof of Theorem $B$. Fix $1<p \leqslant n$, and let $E$ be the corresponding set from Lemma 2.3. Define $u(x)=\operatorname{dist}(x, E)$ for $x$ in the closed unit ball. Then $u$ is continuous in the closed unit ball, $u=0$ in $E$, and $u$ is Lipschitz continuous and hence belongs to $A C L^{p}(B)$. Moreover, $E$ is of positive $p$-capacity and the inequality (3) follows from (4).

REMARKS. (1) The logarithmic term $(\log [1 / \varepsilon])^{p-1}$ in Theorem A can be replaced by, say,

$$
\left(\log \left[\frac{1}{\varepsilon}\right]\right)^{p-1}\left(\log \log \left[\frac{1}{\varepsilon}\right]\right)^{p-1}\left(\log \log \log \left[\frac{1}{\varepsilon}\right]\right)^{p-1}
$$

for all $\varepsilon$ sufficiently small. This is easily seen using the argument of the proof of Theorem A. In fact, any string of iterated logarithms such as above can be used to replace this logarithmic term. So the bound in Theorem A is not best possible. On the other hand, the proof of Lemma 2.3 indicates that one could sharpen Theorem B to allow strings of essentially this type in inequality (3). Since this would cause technical difficulties, we have elected not to do so.

(2) The proof of Theorem A shows that one could make the following modifications to the claim of Theorem A. In the definition of the weak limit, one could only require the given condition for each curve $\gamma$ terminating in $E$ except for curves in a family whose $p$-modulus is zero. The condition $u \in A C L^{p}$ can be replaced by requiring $u$ to be a $p$-precise function. This class, which is larger than $A C L^{p}$, has been studied, for example, in [9], [10] and [16].

(3) One can replace the unit ball $B$ in Theorem $\mathrm{A}$ by any bounded domain $D$ if one replaces the compact set $E$ of positive $p$-capacity by any set $E \subset \partial D$ for which the $p$-modulus of the family of curves joining $E$ to the closure of some open ball in $D$ is positive. For $p=n, D$ uniform and $E$ compact, this is equivalent to $E$ being of positive p-capacity; see $[4,2.6]$.

(4) One often replaces $\left|f^{\prime}(z)\right|$ with $\left|f^{*}(z)\right|$ in questions dealing with analytic (or meromorphic) functions, where $f^{*}(z)=f^{\prime}(z) /\left(1+|f(z)|^{2}\right)$ is the spherical derivative of $f$. Theorem $\mathrm{A}$ is relevant also in this context, as seen by applying it to the function $u(z)=1-1 /(1+|f(z)|)$.

\section{References}

1. D. R. ADAMS and N. G. MeYERs, 'Bessel potentials. Inclusion relations among classes of exceptional sets', Indiana Univ. Math. J. 22 (1973) 873-905.

2. A. Beurling, 'Ensembles exceptionnels', Acta Math. 72 (1940) 1-13.

3. L. CARLESON, 'Sets of uniqueness for functions analytic in the unit disc', Acta Math. 87 (1952) 325-345.

4. D. A. Herron and P. Koskela, 'Quasiextremal distance domains and conformal mappings onto circle domains', Complex Variables 15 (1990) 167-179.

5. J. A. JenkINS, 'On a result of Beurling', Indiana Univ. Math. J. 41 (1992) 1077-1080.

6. P. Koskela, 'Capacity extension domains', Ann. Acad. Sci. Fenn. Ser. A I Math. Dissertationes 73 (1990) $1-42$.

7. V. G. MAZ'JA and V. P. HAVIN, 'Non-linear potential theory', Russian Math. Surveys 27 (1972) 71-148. 
8. Y. Mizuta, 'Existence of various boundary limits of Beppo Levi functions of higher order', Hiroshima Math. J. 9 (1979) 717-746.

9. Y. MizuTA, 'Boundary behaviour of p-precise functions on a half space of $\mathbb{R}^{n}$, Hiroshima Math. J. 6 (1988) 73-94.

10. M. OhtsuKa, 'Extremal length and precise functions in 3-space', Lecture Notes, Hiroshima University, 1973.

11. YU. G. RESHETNYAK, 'Boundary behavior of functions with generalized derivatives Siberian Math. J. 13 (1972) 285-290.

12. M. TsuJI, 'Beurling's theorem on exceptional sets', Tôhoku Math. J. 2 (1950) 113-125.

13. J. VÄISÄLÄ, Lectures on n-dimensional quasiconformal mappings, Lecture Notes in Math. 229 (Springer, Berlin, 1971).

14. E. Villamor, 'Boundary values for some classes of functions', J. London Math. Soc. 45 (1992) 508-518.

15. W. P. ZIEMER, 'Extremal length and p-capacity', Michigan Math. J. 16 (1969) 43-51.

16. W. P. ZIEMER, 'Extremal length as capacity', Michigan Math. J. 17 (1969) 117-128.

Department of Mathematics

University of Michigan

Ann Arbor, MI 48109

USA 